\title{
Optical sensing in medicine
}

\author{
Francesco Baldini
}

Published online: 18 December 2008

(C) Springer-Verlag 2008

Optical sensing has played an important role in medicine since the 1990 s mainly thanks to the invasive capabilities and unique performance of the optical fibres that have allowed measurements inside the human body that would otherwise be impracticable. In recent years, the importance of optics in the biomedical area has increased owing to the advent of nanophotonics, which has opened completely new perspectives.

Monitoring of cell metabolism and intracellular dynamic processes is becoming a reality thanks to the utilisation of natural proteins, which are expressed by specific genes, that exhibit fluorescent and luminescent properties as a function of a well-defined chemical/biochemical parameter. For example, green fluorescein protein (GFP) can be utilised in modified forms to develop cellular biosensors.

Nanoparticles are capable of penetrating cellular membranes and entering the nucleus of the cell. The utilisation of nanoparticles to deliver sensing molecules inside the cell is one of the new frontiers in nanophotonics applied to biomedicine, although their biocompatibility is still under debate. By proper functionalisation and conjugation of suitable chemical transducers or biological recognition elements, these nanocarriers can deliver sensing nanoprobes for a large variety of chemical and biochemical parameters inside cells. Among all the nanoparticles, quantum dots are surely the most used and promising for potential medical diagnostic and basic research, with a large variety of applications thanks to their intrinsic fluorescence and photophysical properties.

\section{F. Baldini $(\bowtie)$}

Department Optoelectronics and Photonics,

Institute of Applied Physics, CNR,

Via Madonna del Piano 10,

50019 Sesto Fiorentino, FI, Italy

e-mail: F.Baldini@ifac.cnr.it
Optical fibres can also be used for cellular monitoring by tapering the fibre end down to tens of nanometers. In this case, the nanoprobe is fixed at the end of the fibre tip and inserted inside cells via micromanipulators. This procedure prevents any problem associated with the uptake and ejection of the nanocarriers from the cells.

Nanoparticles are also being used to develop a new generation of labelling compounds that are able to enhance the optical signal and consequently to improve the sensitivity and the limit of detection of optical biosensors.

Optical biosensors, integrated within an optical biochip, can play a leading role in another emerging area of clinical applications: point of care testing (POCT). In clinical practice, many of the analytes are measured with large and cumbersome equipment located in central laboratories. There is therefore a need for appropriate instruments that are able to efficiently provide results in which the traditional laboratory testing is performed at or near the patient site, usually by nonlaboratory employees (e.g. nurses, respiratory therapists and perfusionists). Because of their miniaturization, low cost and potential for largescale automation, optical biochips can perform analysis more efficiently than currently available laboratory equipment, thus satisfying all the requirements of physicians for a fast and rapid determination of the clinical parameters at the patient's bedside. In contrast to genomics and proteomics, in which thousands of sensing spots are monitored simultaneously by means of fluorescence scanners, many POCT applications often require the measurement of only a limited number of parameters to identify the correct pathology or to monitor the administrated therapy.

All the abovementioned aspects are covered within this special issue. In particular the issue includes two exhaustive reviews on the use of quantum dots for cell monitoring and on the in vivo use of GFP-based indicators for in vivo $\mathrm{pH}$ 
monitoring. Three original papers are concerned with nanophotonics for cell monitoring and signal enhancement, while examples of immunoassays, both label-free and labelbased, for POCT and more generally for clinical applications are given in seven papers. The last original paper describes the design and realisation of a compact optical lab-on-chip device. This last paper, which has more physical than chemical basis, faces the important task of the realisation of the final optical biochip. Its inclusion in this special issue of Analytical and Bioanalytical Chemistry is testament to the importance of the convergence of competences ranging from chemistry and biochemistry to optics, medicine and electronics to achieve the objective of the full development of an optical system for POCT applications. As a matter of fact, one of the consequences of the technology revolution driven by the advent of microand nanotechnology has been the disappearance of the clear-cut division between the different "traditional" sciences (physics, chemistry, biology and medicine) and the birth of new "interdisciplinary fields" such as biotechnology and biophotonics.

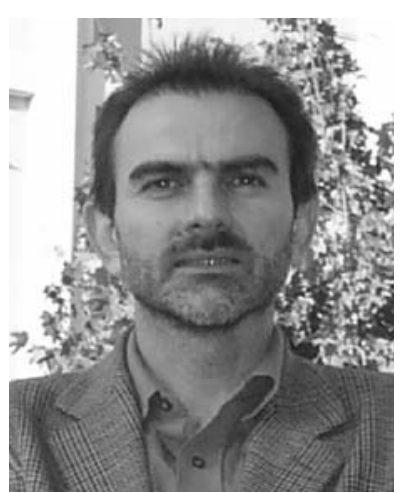

\section{Francesco Baldini}

is a senior researcher at the Institute of Applied Physics of National Council of Research in Florence. His current research activity is devoted to optical sensors for chemical and biochemical parameters. He is the author of more than 70 publications on this subject in international journals, scientific books and international conference proceedings as an invited speaker. $\mathrm{He}$ is a member of the Steering Committee of the Europt(r)ode

Conference on Optical Chemical Sensors and Biosensors and he is also Chairman of the Executive Committee of the Advanced Study Course on Optical Sensors (ASCOS) series. 